\title{
COMPARACIÓN ENTRE LAS ACTITUDES DE LAS PERSONAS CON DISCAPACIDAD INTELECTUAL HACIA LA CONDUCTA SEXUAL Y LAS DE SUS PADRES
}

\author{
Fernández-García, 0. \\ Dpto de Psicología Evolutiva y de la Educación \\ Universitat de València, Valencia, España \\ Olga.Fernandez-Garcia@uv.es \\ Gil-Llario, M.D. \\ Dpto de Psicología Evolutiva y de la Educación \\ Universitat de València, Valencia, España \\ Morell-Mengual, $\mathbf{v}$. \\ Dpto de Psicología Evolutiva y de la Educación \\ Universitat de València, Valencia, España \\ Gil-Juliá, B. \\ Dpto de Personalidad, Evaluación y Tratamientos Psicológicos \\ Universitat de València, Valencia, España
}

Recepción Artículo: 18 enero 2020

Admisión Evaluación: 4 marzo 2020

Informe Evaluador 1: 13 marzo2020

Informe Evaluador 2: 18 marzo2020

Aprobación Publicación: 20 abril 2020

Esta investigación ha sido realizada en el marco del proyecto de investigación "Desarrollo y análisis de la eficacia de un programa de educación afectivo-sexual para prevenir el abuso y mejorar la salud sexual en personas con diversidad funcional intelectual" financiado por el Programa Estatal de l+D+i Orientada a los Retos de la Sociedad del Ministerio de Ciencia e Innovación (Proyecto RTI2018-095538-B-100).

\section{RESUMEN}

Tradicionalmente, las personas con discapacidad intelectual han sido privadas del derecho a expresar su opinión, desestimando su capacidad de reflexión y haciendo prevalecer su mayor dependencia de los adultos de referencia. En este sentido, aunque cada vez hay una mayor conciencia de los derechos de estas personas, siguen presentes los prejuicios de sus padres hacia la sexualidad de este colectivo. Por este motivo, nos proponemos analizar las actitudes hacia la conducta sexual de las personas con discapacidad intelectual que detentan tanto las personas que integran este colectivo como sus padres. Para ello, se administró una escala breve de actitudes hacia la conducta sexual a una muestra de 360 personas con discapacidad intelectual leve o moderada de entre 19 y 55 años y a 100 padres de entre 28 y 83 años. Los resultados revelan que las personas con discapacidad intelectual presentan actitudes significativamente más liberales que sus padres hacia las conductas de besarse, acariciarse $(t=2.423 ; p=.016)$ o realizar prácticas sexuales sin penetración $(t=2.009 ; p=.045)$, pero no hacia la práctica del coito vaginal frente a la que ambos colectivos muestran actitudes semejantes $(t=.252 ; p=.802)$. 


\section{COMPARACIÓN ENTRE LAS ACTITUDES DE LAS PERSONAS CON DISCAPACIDAD INTELECTUAL HACIA LA CONDUCTA SEXUAL Y LAS DE SUS PADRES}

Estos resultados reflejan no solo la importancia de tener en cuenta las actitudes sobre sexualidad de las personas con discapacidad a la hora de elaborar programas de educación afectivo-sexual adaptados a sus características, sino también el valor de proporcionar los conocimientos y habilidades necesarios a los padres.

Palabras clave: actitudes; conducta sexual; padres; discapacidad intelectual

\section{ABSTRACT}

Comparison between the attitudes of people with intellectual disabilities towards sexual behaviour and those of their parents. Traditionally, people with intellectual disabilities have been deprived of the right to express their opinion, dismissing their ability to reflect and making their greater dependence on reference adults prevail. In this sense, although there is an increasing awareness of the rights of these people, the prejudices of parents towards the sexuality of this group are still present. For this reason, we propose to analyse the attitudes towards sexual behaviour of people with intellectual disabilities held by both the people who make up this group and their parents. To do this, a brief scale of attitudes towards sexual behaviour was administered to a sample of 360 people with mild or moderate intellectual disabilities between 19 and 55 years old and 100 parents between 28 and 83 years old. The results reveal that people with intellectual disabilities have significantly more liberal attitudes than their parents towards the behaviours of kissing, caressing $(t=2.423 ; p=.016)$ or engaging in sexual practices without penetration ( $t=2.009 ; p=.045)$, but not towards the practice of vaginal intercourse against which both groups show similar attitudes $(t=.252 ; p=.802)$. These results reflect not only the importance of taking into account the attitudes about sexuality of people with disabilities when developing affective-sexual education programs adapted to their characteristics, but also the value of providing the necessary knowledge and skills to parents.

Keywords: attitudes; sexual behaviour; parents; intellectual disability

\section{INTRODUCCIÓN}

La sexualidad es un aspecto central del ser humano presente a lo largo de todo el ciclo vital, que abarca el sexo, los roles de género, la identidad, el placer y el erotismo (Borawska-Charko, Rohleder y Finlay, 2016). En este sentido, las personas con discapacidad intelectual (DI) experimentan las mismas necesidades y sentimientos sexuales que el resto de adultos (Shewan, Crawley, McKenzie y Quayle, 2014), aunque históricamente han sido privadas de recibir una educación sexual integral que les permita expresar adecuadamente su sexualidad (OMS, 2011). Quizá esto se deba a la consideración sesgada que se tiene de ellos como personas que viven al margen de las experiencias sexuales normativas, carentes de interés sexual (Turner y Crane, 2016; Walker-Hirsch, 2007).

Estos estereotipos aún están muy presentes en los padres de las personas con DI, quienes siempre han evitado proporcionar información sobre sexualidad a sus hijos por temor a despertar su interés sexual que presumiblemente permanecía latente (Walker- Hirsch, 2007). En varios estudios, los padres declaran no sentirse preparados para proporcionar una educación sexual adecuada a sus hijos con DI, y temen que una conversación sobre sexo solo sirva para estimular un comportamiento sexual desmedido e inaceptable socialmente (Pownall, Jahoda y Hastings, 2012; Tamas, Jovanovic, Rajic, Ignjatovic y Prkosovacki, 2019). Esto ocasiona que se muestren reticentes a la hora de permitirles establecer relaciones íntimas o disfrutar de experiencias sexuales propias, ignorando y limitando su expresión sexual y asumiendo el control de la intimidad de sus hijos (Manor Binyamini y Schreiber Divon, 2019). Así, aunque las actitudes están cambiando en las nuevas generaciones de padres (Dupras y Dionne, 2014), algunos siguen tratando la sexualidad de sus hijos como no normativa y fuente de problemas (Manor Binyamini y Schreiber Divon, 2019), y evitan proporcionales los conocimientos necesarios alegando sus limitaciones en el aprendizaje e inmadurez (Ballan, 2012). En este sentido, es innegable que el papel de los padres es primordial en el desarrollo sexual de sus hijos, ya que sus actitudes son predictores muy significativos del funcionamiento sexual y emocional de sus descendientes y determinan la cantidad de información que estos reciben (Haynes, 2016). 
Por lo que respecta a la influencia de ciertas características individuales y sociales en la conformación de las actitudes, varios estudios declaran que tanto la edad como el género son factores determinantes. Los padres y madres de edad más avanzada tienen actitudes menos tolerantes, y los padres tienen actitudes más liberales que las madres de personas con $\mathrm{DI}$, a quienes les resulta emocionalmente difícil pensar que sus hijos son seres sexualmente activos. Así mismo, el nivel de estudios y la cultura o ideología de los padres y madres también contribuye, ya que en culturas muy conservadoras la reproducción es la única finalidad de mantener relaciones sexuales lo que incentiva actitudes más erotofóbicas hacia la sexualidad de las personas con DI (Karellou, 2007; Karellou, 2017).

Por otro lado, aunque hasta el momento son pocas las investigaciones que abordan las actitudes de las personas con DI hacia su sexualidad, los estudios corroboran que este colectivo no solo carece de conocimientos sobre sexualidad (Borawska-Charko, Rohleder y Finlay, 2016; Díaz, Gil, Ballester, Morell, y Molero, 2014; Jahoda y Pownall, 2014), sino que también presenta actitudes más negativas hacia el sexo que las personas sin discapacidad (Bernert y Ogletree, 2013). Concretamente, se ha encontrado que la masturbación y actividades sexuales que requieren cierto grado de intimidad son generalmente aceptadas por las personas con DI, mientras muestran actitudes más conservadoras hacia las relaciones sexuales entre parejas no casadas u homosexuales (Brace, 2007; Karellou, 2017). Los estudios evidencian problemas de moralidad junto a sentimientos positivos hacia las relaciones íntimas, pero continúa siendo difícil para ellos hablar de sexo porque sienten vergüenza y lo perciben como algo grosero y desagradable (Karellou, 2017).

En el caso de las personas con DI, ciertos factores sociodemográficos también influyen en sus actitudes. Los estudios revelan que las mujeres con DI experimentan actitudes más conservadoras hacia su sexualidad que los hombres, llegando a considerar la masturbación como algo negativo para la salud (Bernert y Ogletree, 2013; Chou, Lu y Pu, 2015; Siebelink, de Jong, Taal y Roelvink, 2006). Las mujeres muestran cierta autodeterminación en su comportamiento sexual, pero las percepciones negativas hacia el sexo provocan la abstinencia autoimpuesta. Además, cabe resaltar que las mujeres con DI se ven en doble desventaja, ya que las restricciones hacia la sexualidad de las personas con DI se agravan cuando se combinan con las limitaciones derivadas del hecho de ser mujer (Bernert y Ogletree, 2013).

Por todo lo comentado anteriormente, queda evidenciada la importancia de conocer las actitudes de las personas con DI a la hora de establecer las áreas de intervención de los programas de educación afectivo sexual, y la influencia que las actitudes de los padres ejercen sobre la vivencia de la sexualidad de las personas con DI y sobre la conformación de sus propias actitudes. Por ello, el presente estudio tiene como objetivo analizar las actitudes hacia la conducta sexual de las personas con DI que detentan tanto las personas que integran este colectivo como sus padres. Por un lado, nos proponemos comparar las actitudes de ambos grupos hacia la conducta sexual de las personas con DI en general y hacia diversas prácticas sexuales y, por otro lado, examinaremos la influencia que ejercen ciertos factores sociodemográficos sobre estas actitudes.

\section{MÉTODO}

\section{Participantes}

La muestra del presente estudio está compuesta tanto por personas con DI como por padres de personas que integran ese colectivo. Las 360 personas con DI que han participado se dividen en 180 mujeres y 180 hombres y tienen edades comprendidas entre los 19 y 55 años ( $=39.97$, DT=10.50). El 41,1\% de este grupo de muestra presenta discapacidad intelectual leve, mientras el $58.9 \%$ presenta discapacidad intelectual moderada. Respecto al lugar de residencia, un tercio de la muestra de personas con DI vive en una vivienda tutelada (33.3\%), mientras otro tercio vive en centro residencial (33.3\%) y otro tercio con su familia (33.3\%). El grupo formado por padres de personas con DI, está compuesto por un total de 100 participantes 50 mujeres y 50 hombres con edades comprendidas entre los 28 y los 83 años. La mayoría (56\%) asegura tener el graduado escolar, mientras el 22\% ha acabado los estudios de secundaria, el 10\% tiene estudios superiores y el $12 \%$ no tiene estudios. 


\section{COMPARACIÓN ENTRE LAS ACTITUDES DE LAS PERSONAS CON DISCAPACIDAD INTELECTUAL HACIA LA CONDUCTA SEXUAL Y LAS DE SUS PADRES}

Todos los participantes fueron reclutados mediante muestreo incidental no aleatorio, tratando de compensar diversas características sociodemográficas como la edad, el género y el lugar de residencia. Los criterios de inclusión para las personas con DI fueron: poseer certificado de discapacidad intelectual leve o moderada, contar con suficientes competencias verbales para poder responder los cuestionarios y tener capacidad para dar su consentimiento libremente. Los criterios de inclusión para los padres fueron: ser familiar cercano de una persona con DI, residir en España y tener competencia en lecto-escritura.

\section{Instrumentos}

Cuestionario Ad-Hoc de Información Sociodemográfica. Se trata de un cuestionario que recoge información sobre el género y la edad. En el caso de los padres también pregunta por el nivel de estudios alcanzado (sin estudios, graduado escolar, estudios secundarios, estudios superiores). Incluye dos preguntas específicas sobre el grado de discapacidad (leve o moderada) y el lugar de residencia (vivienda tutelada, centro residencial y unidad familiar) cuando va dirigido a personas con DI.

ASEXDI. Escala de Actitudes hacia la sexualidad en personas con discapacidad intelectual (Gil-Llario, Fernández-García, Castro-Calvo, Caballero-Gascón y Ballester- Arnal, en revisión). Instrumento compuesto por 18 ítems que evalúa las actitudes hacia diversos aspectos de la sexualidad de personas con DI: intimidad, conducta sexual, habilidad para controlar los impulsos sexuales, etc. Se contesta mediante una escala tipo Likert con cinco opciones de respuesta que van desde 1 "completamente en desacuerdo" a 5 "completamente de acuerdo". Para este estudio únicamente se han utilizado 6 ítems relacionados con las conductas sexuales de besarse y acariciarse, prácticas sexuales sin penetración (masturbación y sexo oral) y prácticas sexuales con penetración (coito vaginal). Cada conducta sexual está representada por la puntuación media obtenida del sumatorio de dos ítems. Además de estas puntuaciones medias, también se ha obtenido una puntuación total resultado de la suma de todas las respuestas con un valor entre $3 \mathrm{y}$

Una mayor puntuación indica actitudes más erotofílicas respecto a la sexualidad. A efectos interpretativos se han establecido tres rangos que nos permiten diferenciar entre actitudes conservadoras (de 3 a 6 puntos), moderadas (de 7 a 11 puntos) y liberales (de 12 a 15 puntos).

POS. Escala de percepción sexual para personas con discapacidad intelectual (Scotti, Slack, Bowman y Morris, 1996). Escala compuesta por 28 ítems que evalúa las actitudes positivas o negativas en relación a diferentes comportamientos y prácticas sexuales en parejas del mismo y de diferente sexo. Se responde mediante una escala de respuesta tipo Likert con cinco opciones que van desde 1 "nada aceptable" hasta 5 "muy aceptable". Para este estudio se han seleccionado 6 ítems relacionados con las conductas sexuales de besarse y acariciarse, prácticas sexuales sin penetración (masturbación y sexo oral) y prácticas sexuales con penetración (coito vaginal). Cada conducta sexual está representada por la puntuación media obtenida del sumatorio de dos ítems. Además de estas puntuaciones medias, se ha calculado una puntuación global sumando todas las respuestas con un valor entre 3 y 15. Una mayor puntuación indica actitudes más erotofílicas en relación a las diversas prácticas sexuales. A efectos interpretativos se han considerado tres rangos que diferencian entre actitudes conservadoras (de 3 a 6 puntos), moderadas (de 7 a 11 puntos) y liberales (de 12 a 15 puntos).

\section{Procedimiento}

Para recoger la muestra de personas con DI se contactó con algunos/as psicólogos/as y/o directores/as de diversos centros de atención diurna y residencial para personas con discapacidad y se les proporcionó información sobre el estudio que nos disponíamos a desarrollar. Tras obtener los permisos oportunos de la Junta Directiva se procedió a la evaluación de forma individualizada. En el caso de la muestra de padres de personas con DI fue también el personal de los centros quien se encargó de difundir los cuestionarios entre ellos para que los cumplimentasen en formato lápiz y papel. 


\section{Análisis de datos}

Se emplearon estadísticos descriptivos, ANOVAS y pruebas t de Student, para explorar las actitudes de la muestra hacia la sexualidad de las personas con DI analizando posibles diferencias en función del grupo muestral, género, edad, nivel de estudios, grado de discapacidad y tipo de vivienda. Todos los análisis estadísticos se realizaron con el programa IBM SPSS Statistics 23.

\section{RESULTADOS}

Por lo que respecta a las actitudes hacia la conducta sexual de las personas con DI en general, los resultados extraídos indican que el $36.4 \%$ de las personas con DI poseen actitudes conservadoras mientras el $55.3 \%$ poseen actitudes moderadas y el $8.3 \%$ actitudes liberales. Por su parte, el $49 \%$ de los padres presentan actitudes conservadoras, el otro 49\% actitudes moderadas y solo el $2 \%$ actitudes liberales (ver Figura 1). Así mismo, podemos afirmar que las personas con DI presentan actitudes significativamente más liberales que los padres $(t=3.934$; $\mathrm{p}<.000$ ) hacia la conducta sexual de las personas con DI.

Figura 1. Actitudes hacia la conducta sexual de las personas con DI.

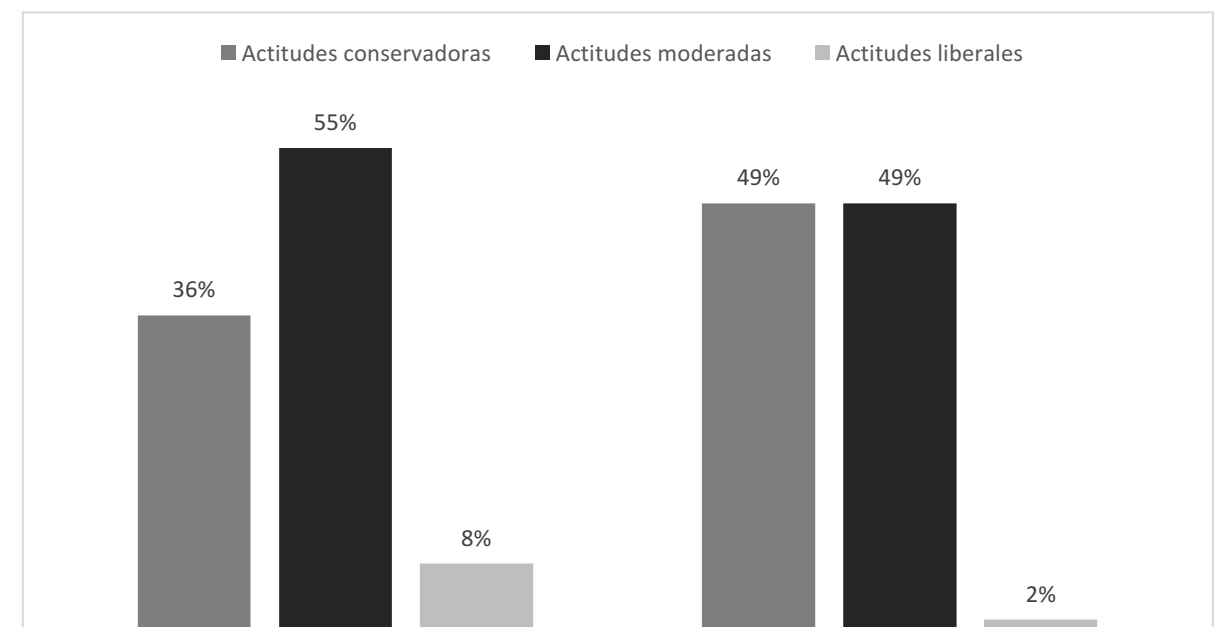

Si analizamos más detalladamente las actitudes de ambos grupos muestrales hacia las diferentes prácticas sexuales evaluadas encontramos que las personas con DI presentan actitudes significativamente más liberales que sus padres hacia las conductas de besarse y acariciarse $(t=2.423 ; p=.016)$ o realizar prácticas sexuales sin penetración ( $t=2.009 ; p=.045)$, pero no hacia la práctica del coito vaginal frente a la que ambos colectivos muestran actitudes semejantes $(t=.252 ; p=.802)$. Así mismo, las prácticas sexuales que reciben mayor apoyo tanto por parte de las personas con DI $(X=2.95, \mathrm{DT}=.98)$ como de los padres $(X=2.68, \mathrm{DT}=.96)$ son aquellas que implican menor grado de intimidad, es decir, los besos y las caricias. Mientras que aquella práctica sexual que implica mayor grado de intimidad (coito vaginal) es la que reciben un menor apoyo en ambos grupos (personas con DI: =2.58, DT=.80; padres: $X=2.56, D T=.73$ ) (ver Figura 2). 


\section{COMPARACIÓN ENTRE LAS ACTITUDES DE LAS PERSONAS CON DISCAPACIDAD INTELECTUAL HACIA LA CONDUCTA SEXUAL Y LAS DE SUS PADRES}

Figura 2. Actitudes hacia las diferentes prácticas sexuales de las personas con DI (puntuaciones medias)

$$
\text { Padres : Personas co } \overline{\mathrm{X}}_{\mathrm{DI}} \overline{\mathrm{X}}
$$

Actitudes hacia los besos y caricias en

personas con DI

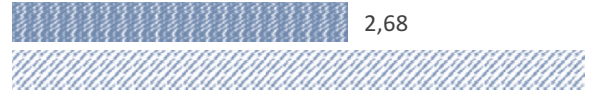

Actitudes hacia las practicas sexuales sin penetración en personas con DI

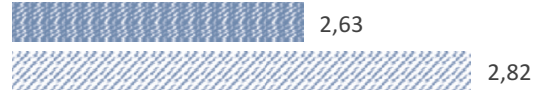

Actitudes hacia las prácticas sexuales con penetración en personas con DI

Cuando analizamos la influencia del género, edad, grado de discapacidad o tipo de vivienda en las actitudes hacia las diversas prácticas sexuales de las personas con DI no se hallan diferencias estadísticamente significativas entre quienes integran este colectivo (ver Tabla 1). En cambio, en el grupo de padres sí que se detectan diferencias estadísticamente significativas entre hombre y mujeres cuando se trata de las actitudes hacia las conductas de besarse y acariciarse $(t=2.168 ; p=.033)$ siendo los hombres quienes presentan actitudes más conservadoras (hombre: $=2.48, \mathrm{DT}=.83$; mujeres: $\mathrm{X}=2.89, \mathrm{DT}=1.04$ ). No se han encontrado diferencias estadísticamente significativas en función de la edad ni del nivel de estudios en las actitudes de los padres, independientemente de la conducta sexual analizada (ver Tabla 2). 
Tabla 1. Actitudes de las personas con DI hacia las diversas conductas sexuales en función del género, grado de discapacidad y tipo de vivienda.

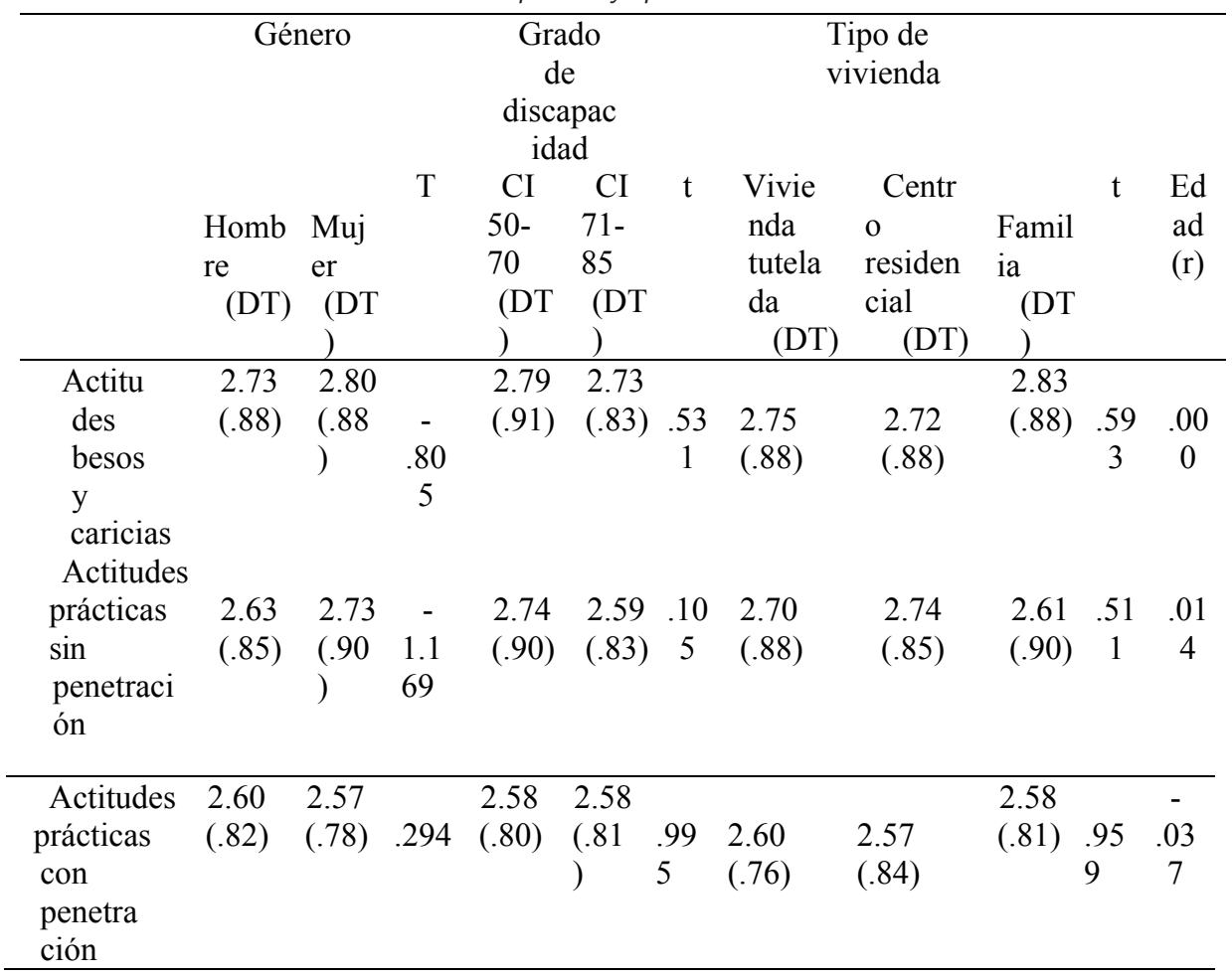


Tabla 2. Actitudes de los padres hacia las diversas conductas sexuales en función del género, edad y nivel de estudios.

\begin{tabular}{|c|c|c|c|c|c|c|c|c|c|c|c|c|c|}
\hline & \multicolumn{2}{|c|}{ Género } & \multirow[b]{2}{*}{$\mathrm{t}$} & \multicolumn{5}{|c|}{ Edad } & \multicolumn{4}{|c|}{$\begin{array}{l}\text { Nivel de } \\
\text { estudios }\end{array}$} & \\
\hline & $\begin{array}{c}\text { Hom } \\
\text { bre }\end{array}$ & $\begin{array}{l}\text { Muje } \\
\text { r } \\
\bar{x} \\
\text { (DT } \\
\text { ) }\end{array}$ & & $\begin{array}{c}28- \\
35 \\
\text { año } \\
\mathrm{s} \\
\bar{x} \\
(\mathrm{D} \\
\mathrm{T})\end{array}$ & $\begin{array}{c}36- \\
51 \\
\text { año } \\
\mathrm{s} \\
\bar{X} \\
(\mathrm{D} \\
\mathrm{T})\end{array}$ & $\begin{array}{c}52- \\
66 \\
\text { año } \\
s \\
\bar{X} \\
(\mathrm{D} \\
\text { T) }\end{array}$ & $\begin{array}{l}67- \\
82 \\
\text { años } \\
\bar{X} \\
\text { (DT } \\
\text { ) }\end{array}$ & $\mathrm{t}$ & $\begin{array}{l}\mathrm{Si} \\
\mathrm{n} \\
\text { Es } \\
\mathrm{t} . \\
\overline{\mathrm{X}} \\
\text { (DT } \\
\text { ) }\end{array}$ & $\begin{array}{l}\text { Gr } \\
\dot{\text { Es }} \\
\mathrm{c.} \\
\bar{X} \\
\text { (DT } \\
\text { ) }\end{array}$ & $\begin{array}{l}\text { E } \\
\text { d. } \\
\text { Se } \\
\text { c. } \\
\bar{x} \\
\text { (DT } \\
\text { ) }\end{array}$ & $\begin{array}{l}\text { Ed } \\
\dot{\mathrm{Su}} \\
\mathrm{p} . \\
\bar{X} \\
\text { (DT } \\
\text { ) }\end{array}$ & $\mathrm{t}$ \\
\hline $\begin{array}{l}\text { Actitud } \\
\text { es } \\
\text { beso } \\
\text { s y } \\
\text { caric } \\
\text { ias } \\
\text { Actitud }\end{array}$ & $\begin{array}{l}2.48 \\
(.83)\end{array}$ & $\begin{array}{l}2.89 \\
(1.04 \\
)\end{array}$ & $\begin{array}{c}2.16 \\
8 *\end{array}$ & $\begin{array}{l}1.50 \\
(.00 \\
)\end{array}$ & $\begin{array}{l}3.00 \\
(.70 \\
)\end{array}$ & $\begin{array}{l}2.5 \\
1 \\
(.90 \\
)\end{array}$ & $\begin{array}{l}2.89 \\
(1.03 \\
)\end{array}$ & $\begin{array}{c} \\
1.7 \\
3\end{array}$ & $\begin{array}{l}2.3 \\
3 \\
(.74 \\
)\end{array}$ & $\begin{array}{l}2.77 \\
(1.01 \\
)\end{array}$ & $\begin{array}{l}2.5 \\
2 \\
(.96 \\
)\end{array}$ & $\begin{array}{l}2.95 \\
(.79)\end{array}$ & $\begin{array}{l}1.1 \\
69\end{array}$ \\
\hline $\begin{array}{l}\text { es } \\
\text { prácticas } \\
\text { sin } \\
\text { penetraci } \\
\text { ón }\end{array}$ & $\begin{array}{l}2.87 \\
(.90)\end{array}$ & $\begin{array}{l}2.78 \\
(.84)\end{array}$ & .513 & $\begin{array}{l}2.25 \\
(.35 \\
)\end{array}$ & $\begin{array}{l}3.25 \\
(.95 \\
)\end{array}$ & $\begin{array}{l}2.8 \\
5 \\
(.79 \\
)\end{array}$ & $\begin{array}{l}2.85 \\
(.92)\end{array}$ & $\begin{array}{c}.69 \\
5\end{array}$ & $\begin{array}{l}3.0 \\
8 \\
(.79 \\
)\end{array}$ & $\begin{array}{l}2.71 \\
(.84)\end{array}$ & $\begin{array}{l}3.0 \\
9 \\
(.89 \\
)\end{array}$ & $\begin{array}{l}2.55 \\
(1.01 \\
)\end{array}$ & $\begin{array}{l}1.6 \\
91\end{array}$ \\
\hline $\begin{array}{l}\text { Actitu } \\
\text { des } \\
\text { prácti } \\
\text { cas } \\
\text { con } \\
\text { penetra } \\
\text { ción }\end{array}$ & $\begin{array}{l}2.62 \\
(.64)\end{array}$ & $\begin{array}{l}2.51 \\
(.81)\end{array}$ & .748 & $\begin{array}{l}3.00 \\
(.70 \\
)\end{array}$ & $\begin{array}{l}2.75 \\
(.86 \\
)\end{array}$ & $\begin{array}{l}2.5 \\
2 \\
(.65 \\
)\end{array}$ & $\begin{array}{l}2.60 \\
(.85)\end{array}$ & $\begin{array}{c}.35 \\
8\end{array}$ & $\begin{array}{l}2.4 \\
1 \\
(.79 \\
)\end{array}$ & $\begin{array}{l}2.53 \\
(.65)\end{array}$ & $\begin{array}{l}2.7 \\
5 \\
(.97 \\
)\end{array}$ & $\begin{array}{l}2.5 \\
(.4 \\
7)\end{array}$ & $\begin{array}{c}.67 \\
9\end{array}$ \\
\hline
\end{tabular}

\section{CONCLUSIONES}

El objetivo de este estudio ha sido analizar las actitudes hacia la conducta sexual de las personas con DI que poseen las propias personas que integran este colectivo y sus padres.

Los resultados obtenidos nos permiten afirmar que las personas con DI presentan actitudes más liberales hacia la expresión de su sexualidad que sus padres. Mientras en la población con DI ya se percibe un cambio actitudinal hacia posturas moderadas- liberales, los padres de personas con DI continúan presentando actitudes conservadoras- moderadas. Estos hallazgos van en la misma dirección que lo expuesto en varios estudios. Bernert y Ogletree (2013) encontraron que, aunque en las mujeres con DI predominaba su percepción negativa hacia el sexo, iban adquiriendo cierta autodeterminación en su comportamiento sexual. Respecto a los padres, Manor Binyamini y Schreiber Divon (2019) afirman que este colectivo continúa negando la sexualidad y las necesidades sexuales de las personas con DI, persistiendo en ellos las actitudes erotofóbicas (Tamas, Jovanovic, Rajic, Ignjatovic \& Prkosovacki, 2019). A todo lo expuesto, Haynes (2016) e investigaciones anteriores (Servais, 2006; Lin, 2009) añaden que las actitudes de los padres actúan como uno de los predictores más reveladores de 
las actitudes de las personas con DI, lo que podría explicar que las actitudes de las personas con DI continúen siendo moderadas.

En nuestro estudio, tanto las personas con DI como los padres expresaron mayor aceptación hacia las conductas sexuales que implican menor grado de intimidad (besos y caricias) que hacia las prácticas que implican mayor intimidad (coito vaginal). A pesar de ello, las personas con DI mostraron actitudes más liberales que los padres hacia todas las prácticas sexuales (besos, caricias, masturbación y sexo oral) a excepción de la práctica del coito vaginal, en la que, aunque se mostraban más liberales no se encontraron diferencias estadísticamente significativas entre ambos grupos. De acuerdo con lo expuesto en varios estudios, las prácticas sexuales que conllevan cierto grado de privacidad, como la masturbación, generalmente son aceptadas por personas con DI mientras que las relaciones sexuales coitales reciben un menor apoyo (Brace, 2007; Karellou, 2017). Estos resultados vendrían justificados por lo comentado por Turner (2012) quien postuló que las personas con DI consideran que la sexualidad no se limita a las relaciones sexuales con penetración. En el caso de los padres puede que las actitudes más erotofóbicas hacia el coito vaginal estén justificadas por ser una práctica sexual asociada a la reproducción y maternidad/paternidad de las personas con DI (Esterle, Sastre y Mullet, 2008; Chou y Lu, 2011; Chu y Lin 2011; Chou, Lu, y Pu 2015).

Por lo que respecta a las diferencias en las actitudes hacia la conducta sexual en función de algunos factores sociodemoiygráficos, hemos encontrado que en las actitudes de las personas con DI no influye ni el género, ni la edad, ni el grado de discapacidad, ni el tipo de vivienda. Esto contrasta con lo obtenido en algunos estudios que afirman que los hombres con DI tienen percepciones más positivas de su sexualidad que las mujeres (Siebelink, de Jong, Taal y Roelvink, 2006; Bernert y Ogletree, 2013; Chou, Lu y Pu, 2015). Por otro lado, las madres sí que muestran actitudes más erotofílicas hacia las conductas sexuales de besarse y acariciarse que los padres, aunque no se han encontrado diferencias en función de otros factores (edad y nivel de estudios). Esto va en contra de lo encontrado en otros estudios que postula que las actitudes de los padres se ven influenciadas por la educación y la edad (Karellou, 2007).

Considerando todo lo expuesto anteriormente, podríamos afirmar que conocer las actitudes de las personas con DI y su entorno puede ser esencial a la hora de planificar y elaborar futuras intervenciones y políticas que tengan una mayor repercusión en la calidad de vida de las personas con DI. Al mismo tiempo, se hace evidente la necesidad de implementar programas de salud sexual no solo dirigidos a las personas con DI sino también a los padres, ya que sus actitudes actúan como uno de los principales predictor del funcionamiento sexual y emocional de las personas con DI.

\section{REFERENCIAS BIBLIOGRÁFICAS}

Ballan, M.S. (2012) Parental perspectives of communication about sexuality in families of children with autism spectrum disorders. J. Autism Dev. Disord. 42(5), 676-684

Bernert, D.J., y Ogletree, R.J. (2013). Women with intellectual disabilities talk about their perceptions of sex. Journal of Intellectual Disability Research, 57, 240-249. doi:10.1111/j.1365-2788.2011.01529.x

Borawska-Charko, M., Rohleder, P. y Finlay, W.M. (2016). The Sexual Health Knowledge of People with Intellectual Disabilities: a Review. Sexuality Research and Social Policy, 14, 393-409.

Brace, E.A. (2007). The sexual lives and identities of women with intellectual disabilities: Exploring the significance of social norms and institutional practices (Unpublished PhD thesis). Newcastle University, Newcastle upon Tyne, UK..

Chou, Y.C. y Lu, Z.J. (2011). Deciding about sterilisation: Perspectives from women with an intellectual disability and their families. Journal of Intellectual Disability Research, 55, 63-74. doi:10.1111/j.13652788.2010.01347.x

Chou, Y.C., Lu, Z.J. y Pu, C.Y. (2015). Attitudes toward male and female sexuality among men and women with intellectual disabilities. Women \& Health, 55, 663- 678. doi:10.1080/03630242.2015.1039183 


\section{COMPARACIÓN ENTRE LAS ACTITUDES DE LAS PERSONAS CON DISCAPACIDAD INTELECTUAL HACIA LA CONDUCTA SEXUAL Y LAS DE SUS PADRES}

Chu, Y.H., y Lin, Y.C. (2011). Sexuality education for parents with a child with intellectual disabilities. Studied in Sexuality, 2, 65-86.

Díaz, I., Gil, M.D., Ballester, A., Morell, V. y Molero, R. (2014). Conocimientos, comportamiento y actitudes sexuales en adultos con discapacidad intelectual. International Journal of Developmental and Educational Psychology, 1(3), 415- 422.

Díaz-Rodríguez, I., Gil-Llario, M.D., Morell-Mengual, V., Salmerón-Sánchez, P., y Ruiz-Palomino, E. (2016). Actitudes hacia la sexualidad: ¿difieren las personas con discapacidad intelectual de la población general? International Journal of Developmental and Educational Psychology, 1(1), 235-242.

Dupras, A., y Dionne, H. (2014). Les préoccupations des parents à l'égard de la sexualité de leur enfant ayant une déficience intellectuelle légère. Sexologies, 23(4), 149-154.

Esterle, M., Sastre, M., Mullet, E. (2008) Judging the acceptability of sexual intercourse among people with learning disabilities: French laypeople's viewpoint. Sex. Disabil. 26, 219-227

Haynes, A. (2016) An investigation into parental attitudes about sexuality and children with intellectual disabilities. Doctoral dissertation, City University of Seattle.

Jahoda, A., y Pownall, J. (2014). Sexual understanding, sources of information and social networks; the reports of young people with intellectual disabilities and their non-disabled peers. Journal of Intellectual Disability Research, 58, 430-441. doi:10.1111/jir.12040

Karellou, J. (2007) Parents' attitudes towards the sexuality of people with learning disabilities in Greece. J. Dev. Disabil., 13(3), 55-72

Karellou, I. (2017) "It Is Only Natural....": Attitudes of Young People With Intellectual Disabilities Toward Sexuality in Greece, Journal of Mental Health Research in Intellectual Disabilities, 10:3, 217-236, D0I: 10.1080/19315864.2016.1278291

Lin C.J. (2009) Intimate relationships of adults with intellectual disabilities: shouldn't or cannot? Gender Education Quarterly, 48, 20-8.

Manor-Binyamini, I. y Schreiber-Divon, M. (2019). Parental Perceptions of the Sexuality of Adolescents with Intellectual Disabilities. Sex Disabil, 37, 599-612. doi: 10.1007/s11195-019-09586-3

Organización Mundial de la Salud (2011). World Report on Disability. World Health Organization, Geneva

Pownall, J.D., Jahoda, A. y Hastings, R.P. (2012) Sexuality and sex education of adolescents with intellectual disability: mothers' attitudes, experiences, and support needs. Intellect. Dev. Disabil. 50(2), 140-154

Servais L. (2006) Sexual health care in persons with intellectual disabilities. Mental Retardation and Developmental Disabilities Research Reviews, 12, 48-56.

Shewan, L., Crawley, R., McKenzie, K. y Quayle, E. (2014). A qualitative exploration of the identities of parents with intellectual disabilities. British Journal of Intellectual Disabilities, 42(1), 19-26.

Siebelink, E.M., de Jong, M.D.T., Taal, E. y Roelvink, L. (2006). Sexuality and people with intellectual disabilities: Assessment of knowledge, attitudes, experiences and needs. Mental Retardation, 44(4), 283-294. doi:10.1352/0047- 6765(2006)44[283:SAPWID]2.0.C0;2

Tamas, D., Jovanovic, N., Rajic, M., Ignjatovic, V., y Prkosovacki, P. (2019). Professionals, parents and the general public: attitudes towards the sexuality of persons with intellectual disability. Sex. Disabil. Doi:10.1007/s1119 5-018-09555-2

Turner G.W. (2012) The social-sexual voice of adults with mild intellectual disabilities: A qualitative case study. PhD Thesis, Widener University, PA, USA.

Turner, G.W. y Crane, B. (2016). Pleasure is paramount: Adults with intellectual disabilities discuss sensuality and intimacy. Sexualities, 19(5-6), 677-697.

Walker-Hirsch, L. (2007). The Facts of Life and More: Sexuality and Intimacy for People with Intellectual Disabilities. Paul H. Brooks, Baltimore 